\title{
Accelerometer for Mobile Robot Positioning
}

\author{
Hugh Liu, Grantham Pang \\ Dept. of Electrical and Electronic Engineering \\ The University of Hong Kong \\ Pokfulam Road \\ Hong Kong \\ Fax: (852)-2559-8738 \\ Tel: (852)-2857-8492 \\ Email: hugh@graduate.hku.hk
}

\begin{abstract}
An evaluation of a low-cost, small sized solid state accelerometer is described in this paper. The sensor is intended for positioning of a mobile robot or platform. Acceleration signal outputted by the sensor is doubly integrated with time which yields the traveled distance. Bias offset drift exhibits in the acceleration signal is accumulative and the accuracy of the distance measurement deteriorates with time due to the integration. Kalman Filter is used to reduce errors caused by random noises. The random bias drift of the accelerometer was found by experiment to be $2.5 \mathrm{mg}$. The accelerometer was moved back and forth three times for a distance of $40 \mathrm{~cm}$ with an acceleration of $8 \mathrm{~m} / \mathrm{s}^{2}$. The final distance error accumulated was $-1.08 \mathrm{~cm}$. The bias drift rate due to temperature was $0.108 \mu \mathrm{g} / \mathrm{s}$ when the accelerometer was placed at room temperature. The results show that the accelerometer could be a viable solution as a short duration distance-measuring device for a mobile robot or platform.
\end{abstract}

\section{INTRODUCTION}

Positioning technologies could broadly be divided into two main streams: relative positioning and absolute positioning. Absolute position means that the currently calculated position does not depend on the previous positions. Example of an absolute positioning system is the Global Positioning System (GPS). The advantage of this system is that there is no accumulation of drift error. However, GPS has the signal blockage problem in outdoors environment. Also, it cannot be used indoors and has relatively low output rate. For a relative positioning system, dead reckoning method is employed to find the position. The angle and distance data are used to find the current position. One of the commonly used relative positioning system is Inertial Navigation System (INS). Dead reckoning positioning with gyros and accelerometers is called inertial navigation. The gyro measures the angular rate and the accelerometer senses the accelerations. Integration of angular velocity with time yields angle data. Distance data could be obtained by double integration of acceleration with time. INS is a self-contained device which requires no external electromagnetic signals. Thus, INS does not have the signal coverage problem found in GPS. Moreover, the data output rate of INS could be much faster than GPS. However, the disadvantage of INS is the bias drift problem. These errors would be accumulated and the accuracy deteriorates with time due to integration. Methods such as Kalman Filter are employed to reduce errors due to the random bias drift.
Compared to an odometer, a 3-axis accelerometer can sense three-dimensional movements while the former can only sense single-axis movements. Also, the data rate of an accelerometer can be much higher than that of an odometer. Moreover, an accelerometer is a self-contained device while an odometer must be fixed to the shaft of some wheels which could be inconvenient in some cases. A solid-state accelerometer also has the advantages of small sized, low cost and being self-contained. Thus, this kind of accelerometer could be a viable solution as a short duration distance-measuring device for mobile robot or platform.

An interesting description of inertial sensors and some innovative applications of the sensors can be found in [1]. Abbott and Powell [2] present a quantitative examination of the impact that individual navigation sensor has on the performance of a navigation system. An evaluation of an Inertial Navigation System for a mobile robot can be found in [3]. Mostov [4] has described about systematic and random errors in an accelerometer. Methods are proposed to reduce the effect of random errors which includes proper modeling of the accelerometer and using feedback system. Reference [5] talks about how to find the bias and scale factor errors by using inertial data and absolute position data.

Below is an outline of this paper. The reduction of random noise of the accelerometer data using Kalman Filter is introduced in Section II. In section III, the accelerometer and the evaluation experiment setups are described. The evaluation methods and experimental results are explained in section IV. In section V, discussions of the results are given. Finally, a conclusion of this paper is given in section VI.

\section{REDUCTION OF RANDOM NOISE USING KALMAN FILTER}

Kalman filter is a commonly used method for random noise reduction and data fusion for positioning applications. In this method, statistical characteristics of a measurement model is used to recursively estimate the required data. A brief introduction of Kalman Filter is given in the following text.

Kalman Filtering is basically a statistical method that combines a knowledge of the statistical nature of system 
errors with a knowledge of system dynamics, as represented by a state space model, to provide an estimate of the state of a system. Any number of unknowns can be included in the states. In a navigation system, we are usually concerned with position and velocity. The state estimate is obtained using a weighting function called the Kalman gain, which is optimized to produce a minimum error variance. [6] A Kalman filter can be used to fuse measurements from multiple sensors and provide both an estimate of the current state of a system and a prediction of the future state of the system. The algorithm of Kalman Filter is show in Fig.1 [7] and the notation of the algorithm is shown in the followings.

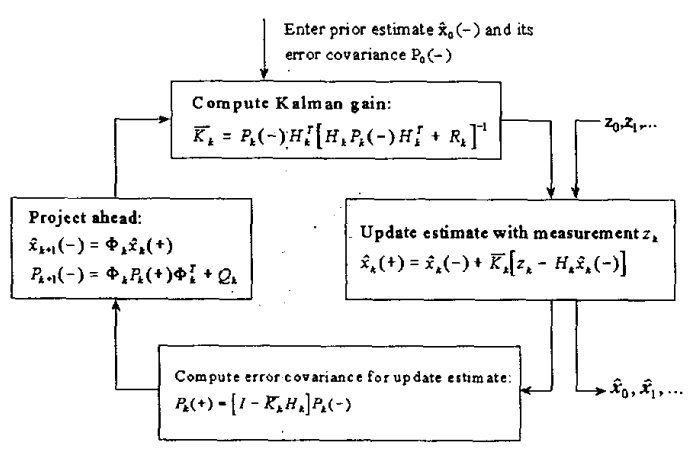

Fig.1. The Kalman Filter algorithm

Notation:

$\mathbf{x}_{\mathbf{k}}$ is the system state

$\mathbf{z}_{\mathbf{k}}$ is the measurement

$\mathbf{w}_{\mathbf{k}}$ is the plant noise with its covariance $\mathbf{Q}_{k}$

$\mathbf{v}_{\mathbf{k}}$ is the measurement noise with its covariance $\mathbf{R}_{\mathbf{k}}$

" $(-)$ " indicates the a priori values of the variables (before the information in the measurement is used).

" $(+)$ " indicates the a posteriori values of the variables (after the information in the measurement is used).

$\bar{K}$ is the Kalman gain.

$\Phi_{k}$ is the transition matrix at time $t_{k}$

$P_{k}$ is the error covariance matrix

$H_{k}$ is the measurement matrix

For reduction of random noises in the accelerometer signal, a process model (Fig.2) with three integrators in cascade was used for processing a single axis of acceleration data. The system parameters are shown in Fig.3 and Fig.4. [8] The power spectral density of the input white noise $W$ is $1\left(\mathrm{~m} / \mathrm{s}^{2}\right)^{2} /(\mathrm{rad} / \mathrm{sec})$, and the sampling time $\Delta t$ equals $1 / 206.6 \mathrm{~s}$. The value of $\mathrm{W}$ was obtained after some experiments to provide better results.

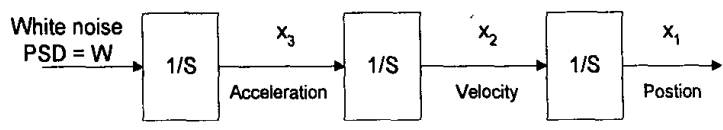

Fig.2. The process model of the accelerometer data for the Kalman Filter

$$
\phi_{k}=\left[\begin{array}{ccc}
1 & \Delta t & \Delta t^{2} / 2 \\
0 & 1 & \Delta t \\
0 & 0 & 1
\end{array}\right]
$$

Fig.3. The state transition matrix for the Kalman Filter

$$
Q_{k}=\left[\begin{array}{lll}
\frac{W}{20} \Delta t^{5} & \frac{W}{8} \Delta t^{4} & \frac{W}{6} \Delta t^{3} \\
\frac{W}{8} \Delta t^{4} & \frac{W}{3} \Delta t^{3} & \frac{W}{2} \Delta t^{2} \\
\frac{W}{6} \Delta t^{3} & \frac{W}{2} \Delta t^{2} & W \Delta t
\end{array}\right]
$$

Fig.4. The error covariance matrix for the Kalman Filter

\section{THE ACCELEROMETER EVALUATION EXPERIMENTAL SETUPS}

\section{A. The Accelerometer}

The accelerometer (interface circuit shown in Fig.5) that has been evaluated is called ADXL202 produced by Analog Device. It is a low cost, low power 2-axis micromachined accelerometer with a measurement range of $\pm 2 \mathrm{~g}\left(19.6 \mathrm{~m} / \mathrm{s}^{2}\right)$. It can measure both dynamic acceleration and static acceleration. The outputs are digital signals whose duty cycles are proportional to the acceleration in each of the two axes. [9] The output can be measured directly with a MCU timer system. This accelerometer is selected for evaluation as a distance measuring sensor due to its small size, low cost and acceptable performance.

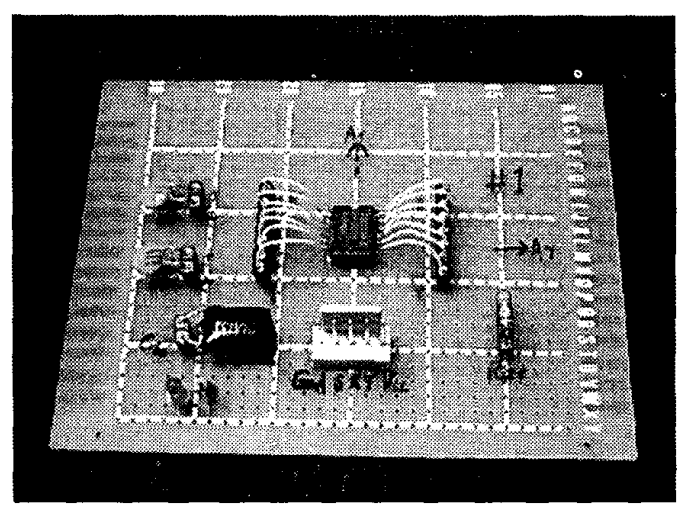

Fig.5. The accelerometer interface board 


\section{B. The Microcontroller and Data Acquisition Board}

The microcontroller used in the data acquisition board is Motorola $68 \mathrm{HCl} 1 \mathrm{~F} 1$. It has 512 bytes of EEPROM, 1024 bytes of RAM, an enhanced 16-Bit timer system, three Input Capture (IC) channels, an enhanced NRZ Serial Communications Interface (SCI).[10] The data acquisition circuit board is shown in Fig. 6 .

The data output by the accelerometer is a $200 \mathrm{~Hz}$ square wave whose duty cycle depends on the acceleration. Input Capture 1 (IC1) pin of the MCU was used to detect the signal from the accelerometer. The MCU would transmit the data to the PC via the Serial Communication Interface (SCI). A Visual Basic program was used at the $\mathrm{PC}$ to receive and save the data to the hard disk. A C program was written to process large amount of accelerometer data. The recorded data is downsampled by averaging the acceleration data within each downsampling period. The downsampled data was then stored in a file which could be plotted using MATLAB. For the results obtained, the data was averaged every twenty-five seconds to give a downsampled data.

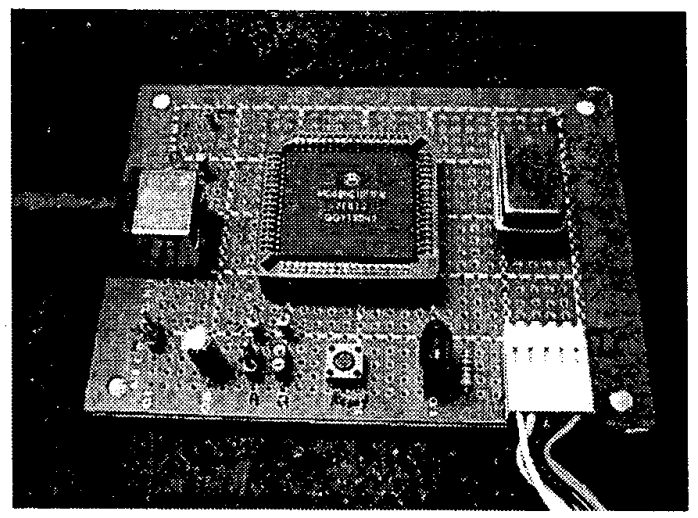

Fig.6. The data acquisition board with the microcontroller

\section{The Sony Robot Arm}

The robot arm used to move the accelerometer is a Sony SRX-410 High Speed Assembly Robot. It was designed for high assembling speed and high performance industrial applications. The system consists of a robot arm, a controller, a panel and a PC for programming the robot arm. Fig. 7 shows a photograph of the accelerometer evaluation hardware setup. The block diagram of the experimental setup is shown in Fig.8.

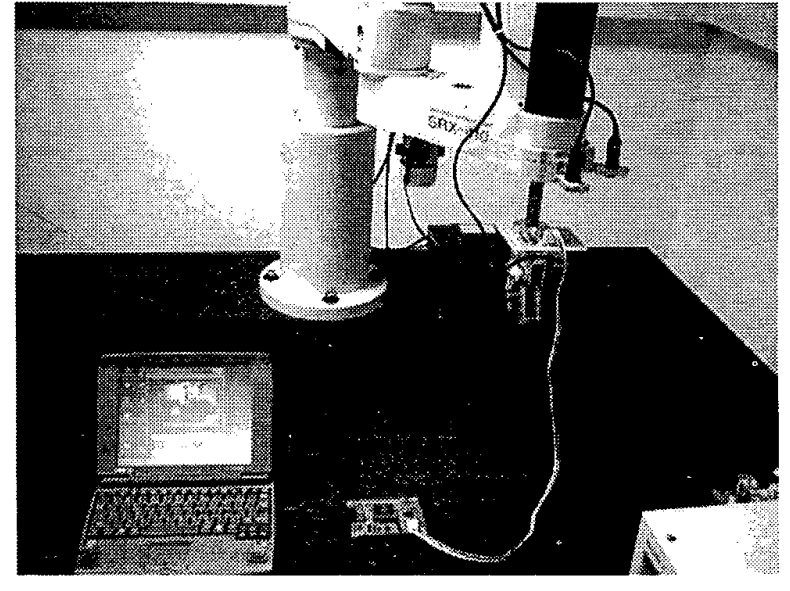

Fig.7. The accelerometer evaluation experiment hardware setup

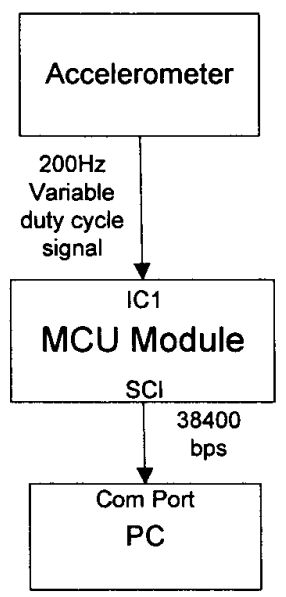

Fig. 8. Accelerometer data acquisition block diagram

\section{EVALUATION METHODS AND EXPERIMENTAL RESULTS}

\section{A. 14-hour of Stationary Accelerometer Data}

Fourteen hours of stationary accelerometer data was taken in order to study the effect of temperature on the bias drift. The recorded data occupied 81.3 Mbytes of hard disk space. The data was processed to give the acceleration readings which was then downsampled for plotting using MATLAB.

The duty cycle of the accelerometer output is proportional to the acceleration. The microcontroller was used to measure the duty cycle using the timer system. The timer counts was converted to ASCII and then sent to the PC for recording. The data was processed according to Fig.9.

Fig.10 and Fig.11 show the 14-hour acceleration data without and with Kalman filter processing respectively. The thermal 
bias drift rate of the accelerometer placed at room temperature was found by this experiment to be $0.108 \mu \mathrm{g} / \mathrm{s}$.

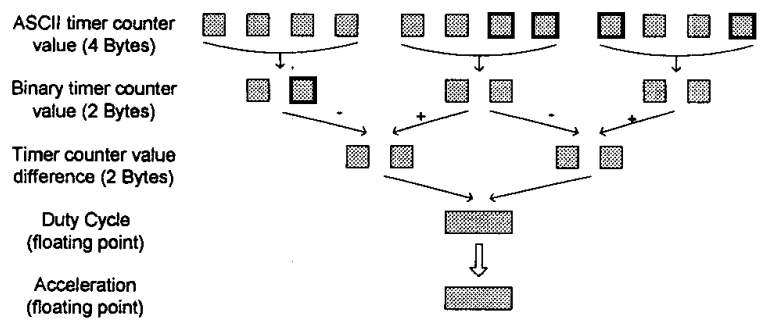

Data structures involved in calculating the acceleration

Fig.9. Data processing diagram for the acceleration data

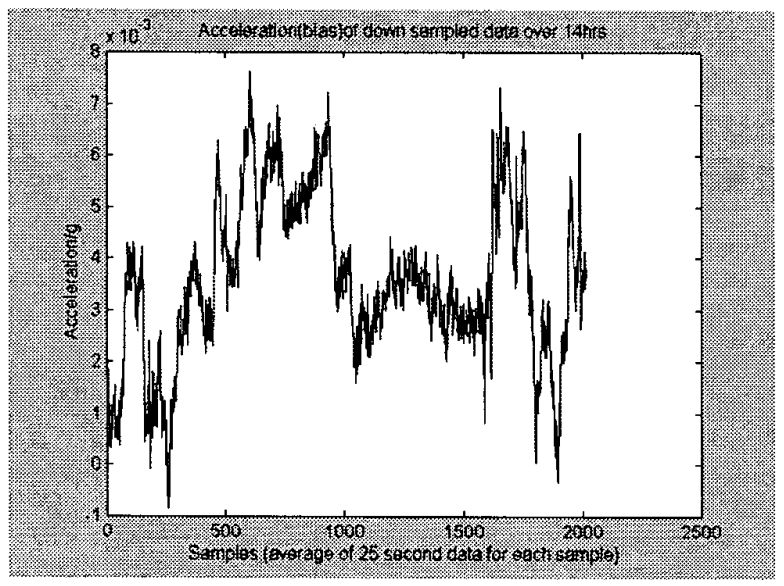

Fig.10. Acceleration data in 14 hours without Kalman Filter processing

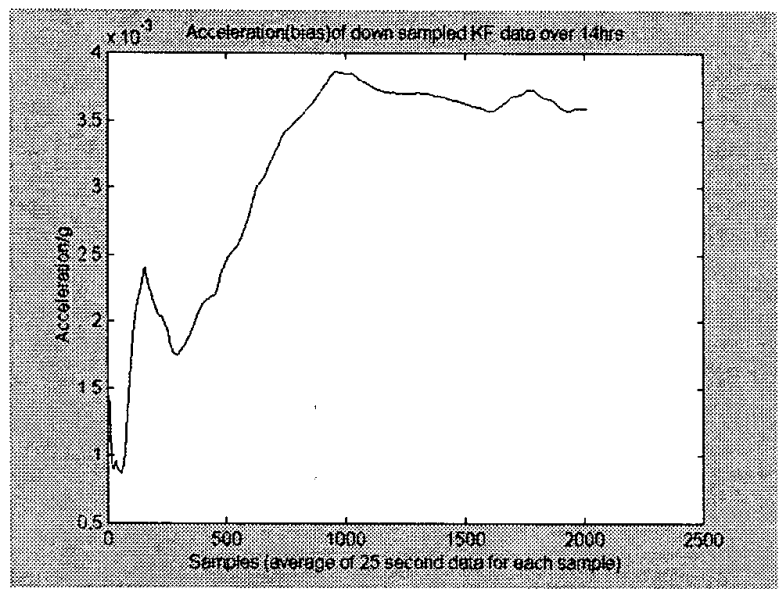

Fig.11. Acceleration data in 14 hours with Kalman Filter processing

\section{B. Experiments Carried Out Using Sony Robot Arm}

Experiments for the accelerometer evaluation were carried out using the Sony Robot Arm. Twenty three sets of experiment with different velocity and acceleration combinations were conducted. The velocities range from 0 $\mathrm{m} / \mathrm{s}$ to $1 \mathrm{~m} / \mathrm{s}$ and the accelerations ranges from $0 \mathrm{~m} / \mathrm{s}^{2}$ to $10 \mathrm{~m} / \mathrm{s}^{2}$. Three different sets of results with low, moderately high and high acceleration is presented in the following subsections.

\section{(1) Low Acceleration $\left(a=3 \mathrm{~m} / \mathrm{s}^{2}\right.$ )}

Figures 12-15 show the results of the experiment with acceleration at $3 \mathrm{~m} / \mathrm{s}^{2}$ which is quite low and with velocity at $0.3 \mathrm{~m} / \mathrm{s}$. The accelerometer was moved from left to right and vice versa for a distance of $40 \mathrm{~cm}$. Such motions were repeated for three times. Figures 12 and 13 show the acceleration data without and with Kalman Filtering. Due to the random bias drift problem, the acceleration data was divided into regions for different bias reductions. The biases in the first seven regions were manually tuned to optimize the accuracy. The last six regions were not calibrated for comparison purpose. These calibrations also helped to estimate the random bias drift of the accelerometer. The values of the manually tuned biases are plotted in Fig.16. Fig. 14 shows the velocity which was calculated by integrating the acceleration data with time. Only the first two velocity cycles are calibrated with manually tuned acceleration biases. Fig. 15 shows the distance which was calculated by integrating the velocity data with time. Only the first two distance cycles are calibrated with manually tuned acceleration biases. This graph shows the affection of the random bias drift on the distance data after double integration of the accelerometer data.

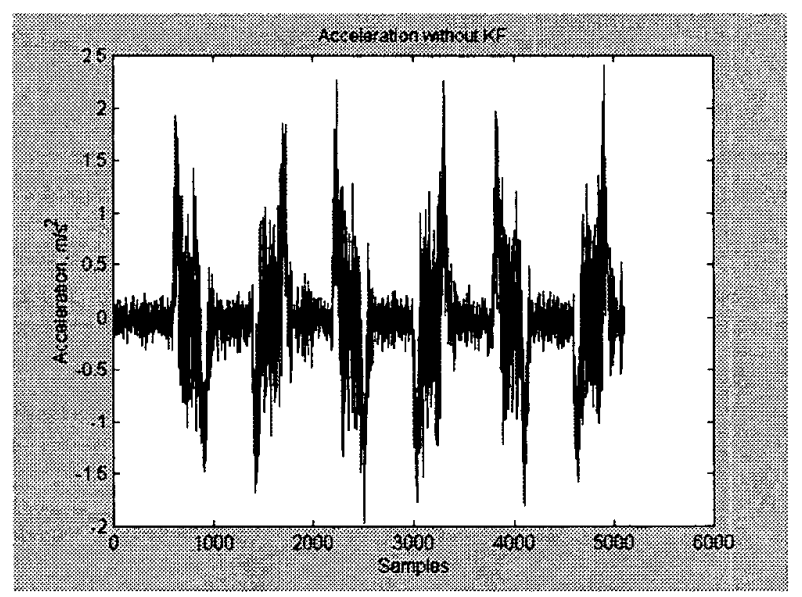

Fig.12. Data of acceleration at $3 \mathrm{~m} / \mathrm{s}^{2}$ without Kalman Filtering 


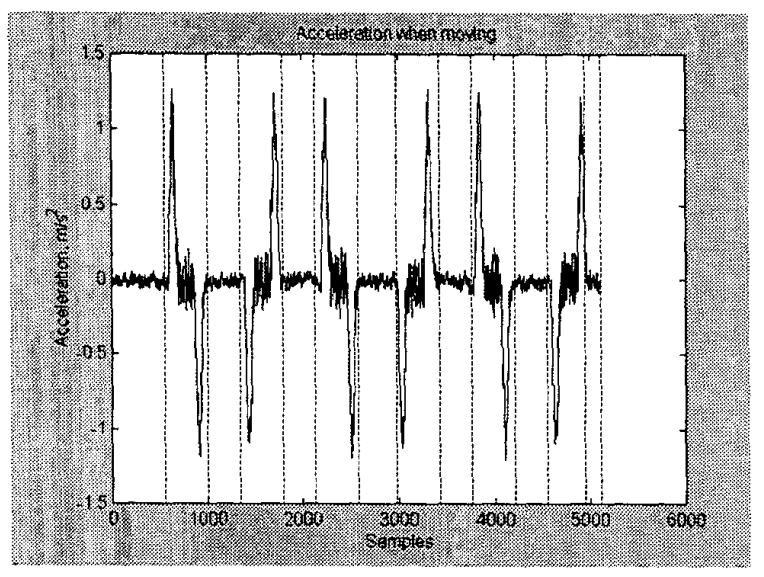

Fig.13. Data of acceleration at $3 \mathrm{~m} / \mathrm{s}^{2}$ after Kalman Filtering was partitioned and calibrated with different biases

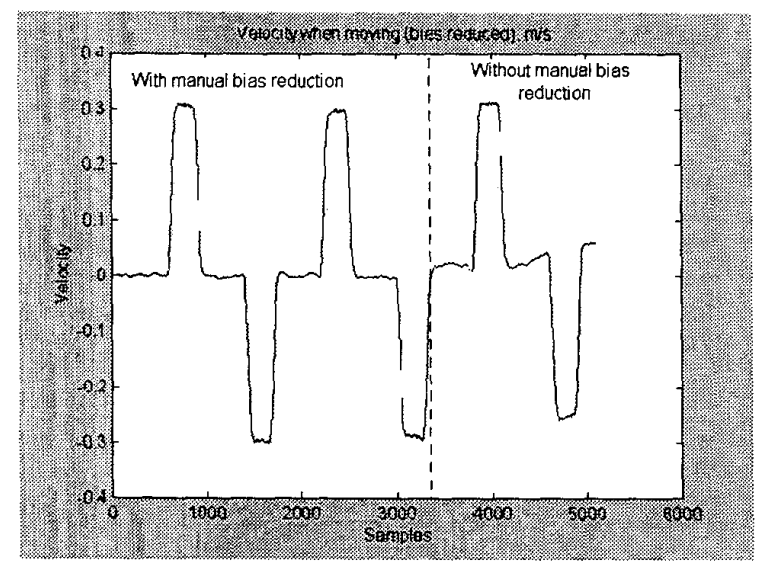

Fig.14. Velocity with and without manually tuned acceleration biases reduction (only first two velocity cycles are calibrated)

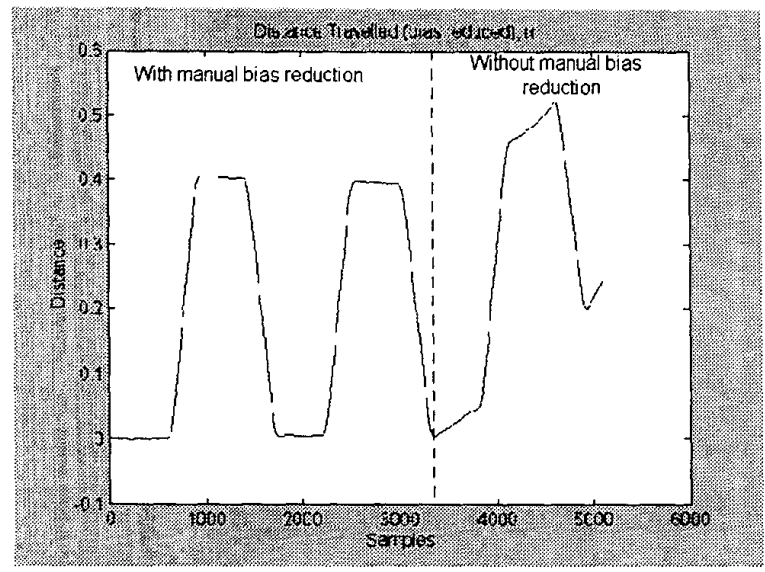

Fig. 15. Distance traveled with and without manually tuned acceleration biases reduction (only the first two distance cycles are calibrated)

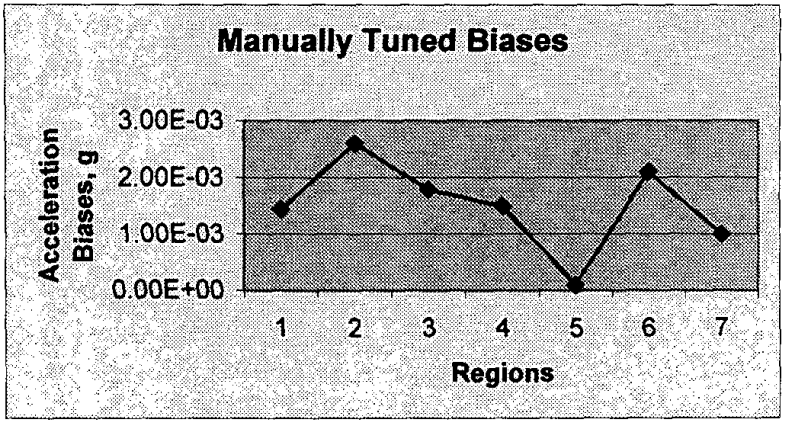

Fig.16. Manually tuned biases for the first seven regions of the acceleration data

\section{(2) Moderately High Acceleration $\left(a=8 \mathrm{~m} / \mathrm{s}^{2}\right)$}

Figures 17-20 show the results of the experiment with acceleration at $8 \mathrm{~m} / \mathrm{s}^{2}$ which is quite high and with velocity at $0.8 \mathrm{~m} / \mathrm{s}$. Again, the accelerometer was moved from left to right and vice versa for a distance of $40 \mathrm{~cm}$. Such motions were repeated for three times. The acceleration was calibrated with a constant bias to reduce the zero offsets.

Fig.17 shows the acceleration data without Kalman Filter processing. This has resulted in a signal disturbed with random noises. A clearer shape of the signal obtained by using Kalman Filter is shown in Fig.18. The integrated velocity is shown in Fig.19 and the integrated distance is shown in Fig.20. The final distance was found to be $-1.08 \mathrm{~cm}$ while the actual final distance should be zero.

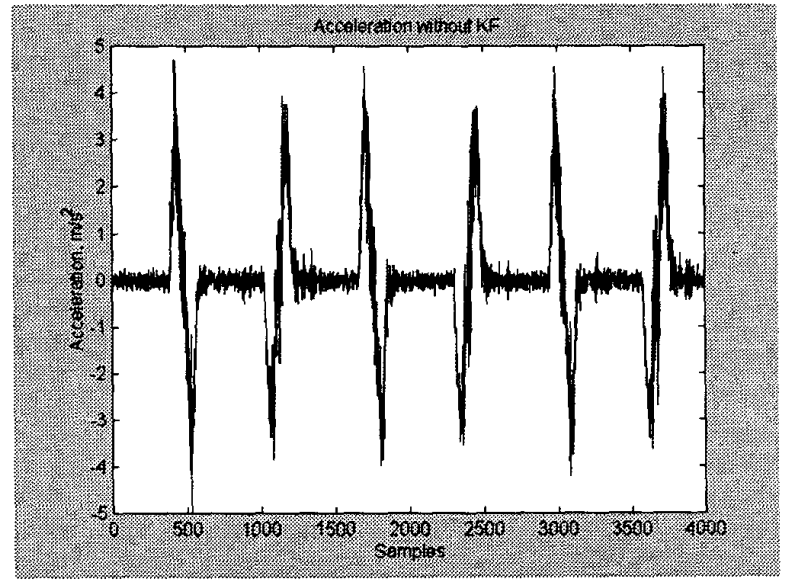

Fig. 17. Acceleration results for acceleration of $8 \mathrm{~m} / \mathrm{s}^{2}$ and velocity of $0.8 \mathrm{~m} / \mathrm{s}$ without Kalman Filter processing 


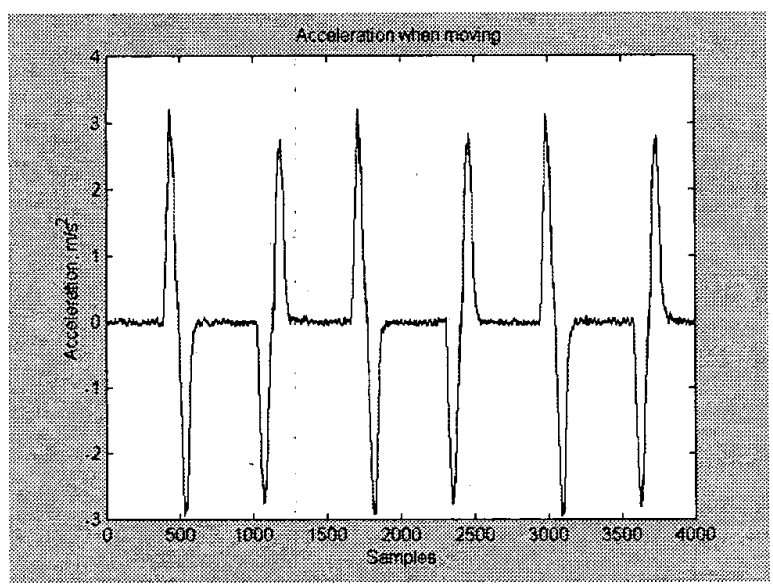

Fig. 18. Acceleration results for acceleration of $8 \mathrm{~ms}^{-2}$ and velocity of $0.8 \mathrm{~ms}^{-1}$ with Kalman Filter processing

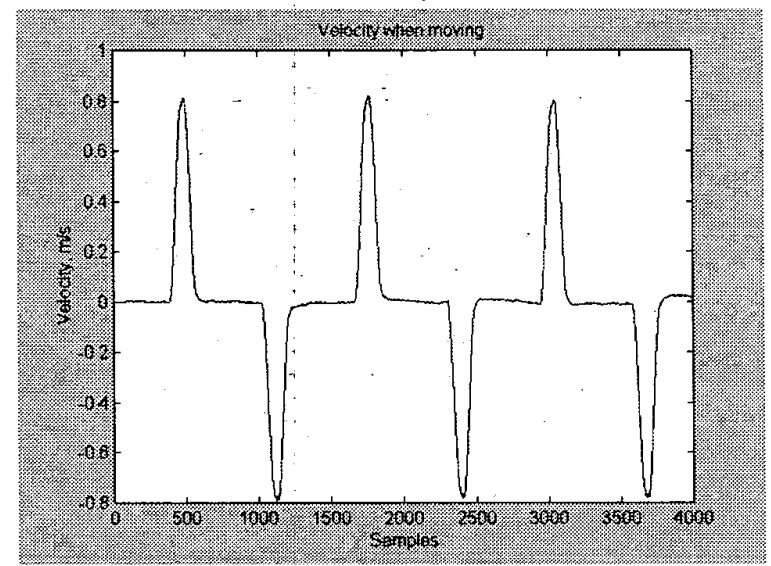

Fig. 19. Integrated velocity results for acceleration of $8 \mathrm{~ms}^{-2}$ and velocity of $0.8 \mathrm{~ms}^{-1}$

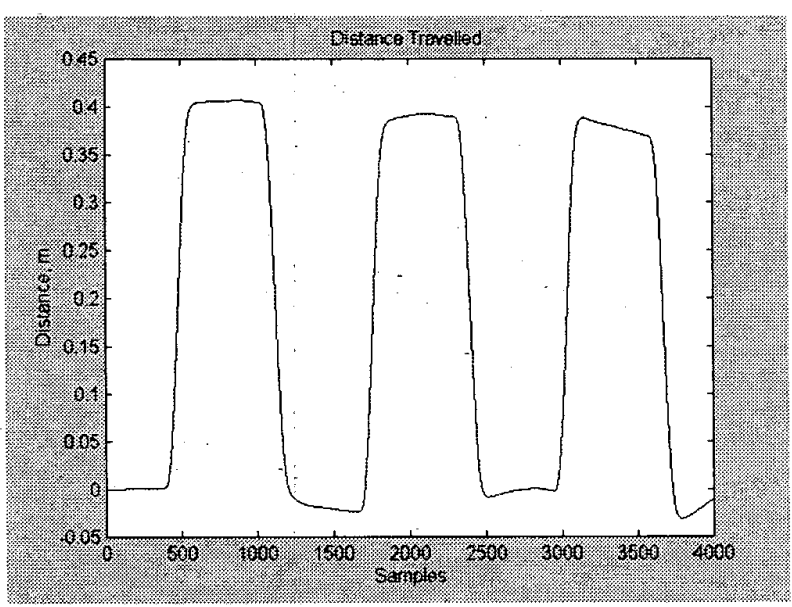

Fig. 20. Integrated position results for acceleration of $8 \mathrm{~ms}^{-2}$ and velocity of $0.8 \mathrm{~ms}^{-1}$

\section{(3) High Acceleration $\left(a=10 \mathrm{~m} / \mathrm{s}^{2}\right)$}

Figures 21-24 show the results of the experiment with acceleration equals $10 \mathrm{~m} / \mathrm{s}^{2}$ which is relatively high and velocity equals $1 \mathrm{~m} / \mathrm{s}$. The accelerometer was moved from left to right and vice versa for a distance of $40 \mathrm{~cm}$. Such motions were repeated for eight times. The acceleration was calibrated with a constant bias to reduce the zero offsets.

Figure 21 and 22 show the acceleration without and with Kalman Filter processing respectively. The velocity and the distance are shown in Fig.23 and Fig.24 respectively. The final distance was found to be $+1.55 \mathrm{~cm}$.

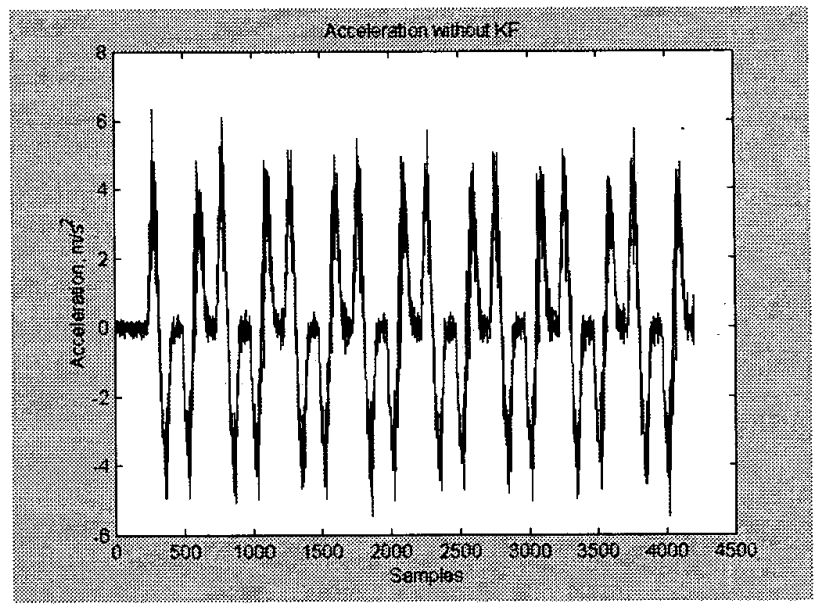

Fig. 21 Acceleration results for acceleration of $10 \mathrm{~ms}^{-2}$ and velocity of $1 \mathrm{~ms}^{-1}$ without Kalman Filter processing

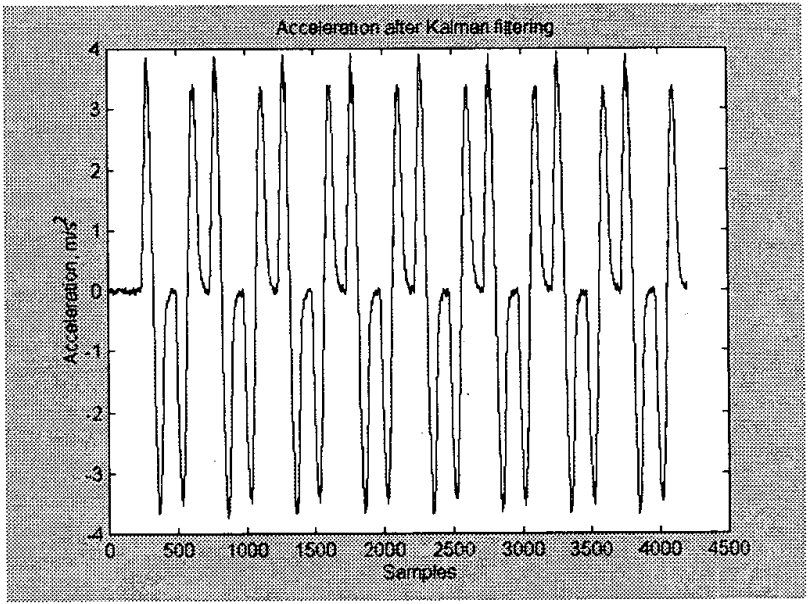

Fig.22 Acceleration results for acceleration of $10 \mathrm{~ms}^{-2}$ and velocity of $1 \mathrm{~ms}^{-1}$ with Kalman Filter processing 


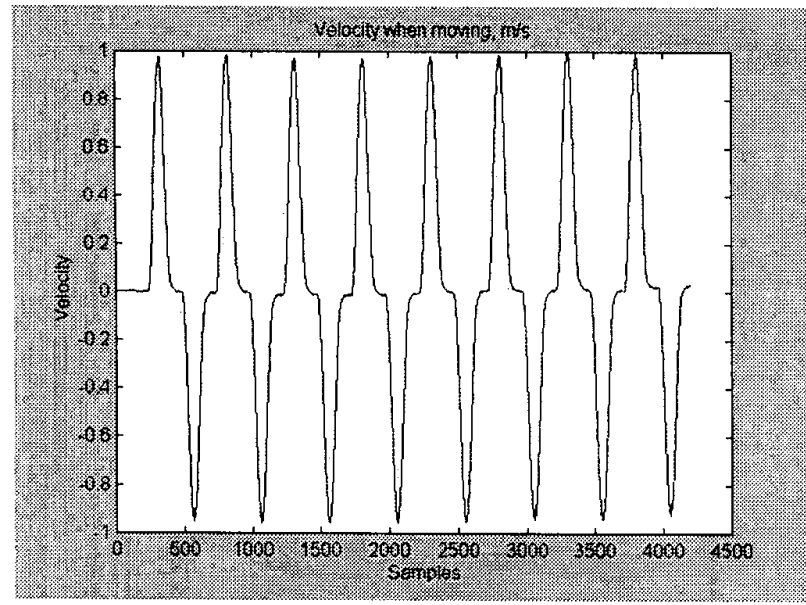

Fig.23 Velocity results for acceleration of $10 \mathrm{~ms}^{-2}$ and velocity of $1 \mathrm{~ms}^{-1}$

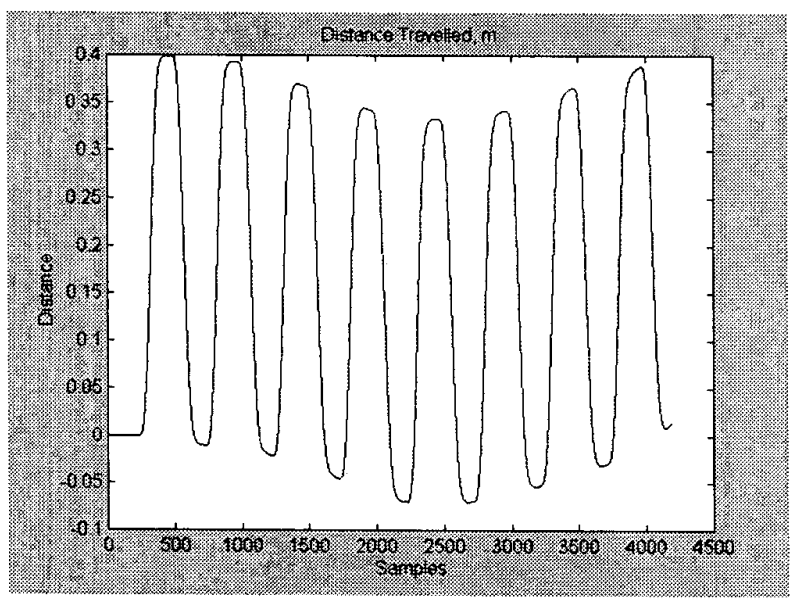

Fig. 24 Distance results for acceleration of $10 \mathrm{~ms}^{-2}$ and velocity of $1 \mathrm{~ms}^{-1}$

\section{DISCUSSIONS}

\section{A. Behavior of the 14-hour Stationary Accelerometer Data}

From the result shown in Fig. 11, it is observed that the bias or zero offset of the accelerometer generally increased after powering up. After about seven hours, the bias settled down to fairly stable values. The results are due to the thermal bias drift of the accelerometer. The internal temperature of the sensor increases when it is warming up. The thermal bias drift rate when the accelerometer is placed at room temperature is found in this evaluation to be $0.108 \mu \mathrm{g} / \mathrm{s}$. If these bias drifts due to the temperature are not compensated, the results would be affected after operating for long duration. Compensation methods can be done by using low cost temperature sensing IC and crystal oven. [11]

\section{B. Experiments Carried Out Using Sony Robot Arm}

\section{(1) Random Bias Drift}

From the graph of manually tuned biases (Fig.16), the range of the biases deviations is about $2-3 \mathrm{mg}$ which is fairly large. Better navigational grade accelerometer has about $0.1 \mathrm{mg}$ random bias drift. The velocity error and the position error built up could be calculated by the equations below [12]:

Velocity error $=0.589 \mathrm{~m} / \mathrm{s}$ per $\mathrm{mg}$ per $\min$

Position error $=17.66 \mathrm{~m}$ per $\mathrm{mg}$ per $\mathrm{min}^{2}$

Thus, for a bias error of $2 \mathrm{mg}$, the velocity errors built up in one minute is $1.178 \mathrm{~m} / \mathrm{s}$. Moreover, the position error built up in one minute for a bias error of $2 \mathrm{mg}$ is $35.32 \mathrm{~m}$. Thus, if the random bias could be modeled properly, the accuracy in distance measurement can be greatly improved.

\section{(2) Relation Between Magnitude of Acceleration and Accuracy in Distance Measurement}

From the distance graphs of Fig.20 and Fig.24, distortions are observed which were due to the random biases of the accelerometer. The random bias drift error is one of the major sources of the positioning error. When the acceleration is higher, the errors caused by the random bias are less significant.

The integrated final distance was found to be $-1.08 \mathrm{~cm}$ and $+1.55 \mathrm{~cm}$ when the accelerations were $8 \mathrm{~m} / \mathrm{s}^{2}$ and $10 \mathrm{~m} / \mathrm{s}^{2}$ while the actual final distances should both be zero. Thus, the results are quite close to the ideal ones. These good results were due to the relatively large acceleration imposed on the accelerometer. As the accelerometer can measure up to $2 \mathrm{~g}$ which is $19.6133 \mathrm{~m} / \mathrm{s}^{2}$ of acceleration, the applied acceleration which are $8 \mathrm{~m} / \mathrm{s}^{2}$ and $10 \mathrm{~m} / \mathrm{s}^{2}$ are relatively large. When compared with Fig.14, the distortions in distance measurement for higher accelerations are less. This means when the acceleration is higher, the errors caused by the random biases are less significant.

\section{CONCLUSIONS}

The experimental results have provided a useful evaluation of a low-cost solid state accelerometer. The performance of the accelerometer is shown to be acceptable as a short duration distance-measuring device for mobile platform or robot. Such an accelerometer could be a self-contained sensor to give a low cost and small-sized distance-measuring device for mobile robot, platform or vehicle. It can be combined with gyroscope and odometer to form a dead reckoning positioning system for a mobile robot or platform. Further research would be on the proper modeling of the accelerometer in order to reduce the effect of random bias drift.[4] Moreover, the combination of digital compass, gyroscope, odometer and beacon signals with the accelerometer would be studied. 


\section{REFERENCES}

[1] C. Verplaetse, "Inertial Proprioceptive Devices: SelfMotion Sensing Toys and Tools," IBM System Journal, Vol.35, Nos3\&4, 1996.

[2] Eric Abbott, David Powell, "Land-Vehicle Navigation Using GPS," Proceedings of IEEE, Vol.87, No.1, 1999.

[3] Billur Barshan, Hugh F. Durrant-Whyte, "An Inertial Navigation System for a Mobile Robot," Proc. International Conference on Intelligent Robots and Systems, Yokohama, Japan, 1993.

[4] Kirill Mostov, "Inertial Sensor Documentation", Web Page of PATH, UC Berkeley, http://www.path.berkeley.edu/ webed/sensor/papers.html.

[5] Kirill Mostov, "Method for correction of systematic inertial sensor error" Web Page of PATH, UC Berkeley, http://www.path.berkeley.edu/ webed/sensor/papers.html.

[6] Elliott D. Kaplan, Understanding GPS: Principle and Applications. Artech House, Boston, 1/e, 1996, p.39

[7] Robert Grover Brown, Patrick Y.C. Hwang, Introduction to Random Signals and Applied Kalman Filtering. John Wiley \& Sons, 3/e, 1997, p.219

[8] Robert Grover Brown, Patrick Y.C. Hwang, Introduction to Random Signals and Applied Kalman Filtering. John Wiley \& Sons, 3/e, 1997, p.232

[9] Analog Devices, ADXL202 data sheet. 1998

[10] Motorola, 68HC11F1 Technical Data. 1995

[11] Charles Kitchin, Understanding Accelerometer Scale Factor and Offset Adjustments. Analog Devices.

[12] Anthony Lawrence, Modern Inertial Technology. Spring- Verlag, 1993, p.28. 